journal club

\title{
Lässt sich die Überlebenszeit vorhersagen?
}

Fragestellung: In dieser Arbeit sollte untersucht werden, ob klinische Symptome sowie das Testen des autonomen Nervensystems eine zuverlässige Vorhersage der Überlebenszeit von Patienten mit Multisystematrophie (MSA) erlauben.

Hintergrund: Die Multisystematrophie wird klinisch in den Parkinson-Typ (MSA-P) und zerebellären Typ (MSA-C) aufgeteilt, je nachdem, ob die Parkinson-Symptomatik mit Bradykinese, Rigor und weniger häufig Tremor im Vordergrund steht, oder die Gangunsicherheit und die generalisierte Ataxie neben dem Parkinsonismus vorliegen. Es ist bekannt, dass Patienten mit MSA neben diesen zerebellären und Parkinson-typischen Symptomen auch schwere Störungen des autonomen Nervensystems sowie des sudomotorischen Systems aufweisen. Sehr viele Patienten zeigen insbesondere Störungen der Orthostase, des Urogenitalsystems und der gastrointestinalen Funktionen. In dieser Arbeit sollte untersucht werden, ob bei Patienten mit MSA-Typ P oder C basierend aus einer besonderen Konstellation der Störungen des autonomen Nervensystems, des Beginns der Erkrankung oder des Geschlechtes eine Prädiktion bezüglich der Überlebenszeit möglich ist.

Patienten und Methodik: Die Studie schloss MSA-Patienten ein, bei denen nach MSA-P und MSA-C unterschieden wurde und sehr sorgfältig die klinische Symptomatologie sowie durch präzises Befragen sämtliche Funktionen des autonomen Nervensystems erfasst wurden. Zudem wurden Untersuchungsergebnisse voruntersuchender Neurologen herangezogen. Wenn eine frühere Diagnose wie olivopontozerebelläre Atrophie oder striatonigrale Degeneration oder Shy-Drager-Syndrom gestellt worden war, wurde dies als MSA reklassifiziert. Alle Patienten unterliefen eine standardisierte autonome Testung mit quantitativen Untersuchungen des sudomotorischen Axonreflexes, der Herzfrequenz und deren Variabilität, des Blutdrucks unter Valsalva-Manöver sowie einen Tilt-Test. Dazu wurde ein validierter Composite Autonomic Severity Score für alle Patien-

Coon, EA, Sletten, DM, Suarez, $\mathrm{MD}$ et al. Clinical features and autonomic testing predict survival in multiple system atrophy. BRAIN 2015; 138: $3623-31$ ten berechnet. Unter orthostatischer Hypotension wurden Patienten subsummiert, die bei Aufstehen aus dem Liegen ein Absinken des sys- tolischen Blutdruckes von über $30 \mathrm{mmHg}$ und des diastolischen Blutdrucks um mehr als $15 \mathrm{mmHg}$ aufwiesen. MRT-Bilder wurden nur dann herangezogen, wenn sie ein Neuroradiologe begutachtet hatte. MSA wurde immer dann diagnostiziert, wenn zerebelläre Atrophie, hot cross bun sign, Hyperintensität der mittleren zerebellären Peduncel, putaminale Hypointensität und Hyperintensität entlang des Putamens gesehen wurden.

Ergebnisse: Insgesamt wurden 685 Patienten in die Studie aufgenommen, von denen 594 eine wahrscheinliche und 91 eine mögliche MSA aufwiesen. Den MSA-P-Sub-Typ zeigten $430 \mathrm{~Pa}-$ tienten (63\%). Die Patienten waren bei Krankheitsbeginn zwischen 34 und 86 Jahre alt. Von den 685 untersuchten Patienten waren zum Zeitpunkt der Evaluation 541 verstorben. Von 36 Patienten wurde eine neuropathologische Post-mortem-Untersuchung gefunden, die bei allen die Verdachtsdiagnose MSA bestätigte. Bei Patienten mit MSA-C waren die ersten Symptome im Durchschnitt im Alter von 48,4 Jahren aufgetreten, bei dem MSA-Typ-P-Patienten erst mit 62,3 Jahren. Die mittlere Krankheitsdauer bis zum Tod betrug 7,51 Jahre, wobei die Zeit vom Stellen der Diagnose bis zum Tod im Schnitt 3,33 Jahre dauerte. Es gab keinen Unterschied bezüglich der Überlebenszeit zwischen den beiden Subtypen der MSA. Meist (61\%) hatten die Patienten zuerst den Parkinsonismus oder das zerebelläre Bild, bevor sie Störungen des autonomen Nervensystems entwickelten. Der zeitliche Ablauf, ob Patienten zuerst motorische oder Störungen des autonomen Nervensystems aufwiesen, beeinflusste die Überlebenszeit nicht.

Mithilfe statistischer Analysen konnte gezeigt werden, dass eine verkürzte Lebenserwartung für die Patienten galt, die in den ersten drei Jahren nach Erkrankungsbeginn gestürzt waren, Störungen der Blasenfunktion aufwiesen, Notwendigkeit der Katheterisierung drei Jahre nach Symptombeginn, orthostatische Intoleranz innerhalb eines Jahres nach Symptombeginn oder zum Krankheits- und Symptombeginn in fortgeschrittenem Alter waren. Zudem wiesen die Patienten, die im Composite Autonomoc Severity Score besonders schlecht abschnitten, eine schlechte Lebenserwartung auf.

Schlussfolgerungen: Die Autoren schlossen aus ihren Beobachtungen, dass bei Vorliegen oben genannter Risikofaktoren eine nennenswert beeinträchtigte Lebenserwartung gegeben ist.

\section{- Kommentar von Heinz Reichmann, Dresden}

\section{Die Ergebnisse erlauben eine gute Abschätzung der Prognose}

Diese Arbeit ist von großer Qualität, weil sie an einer hohen Zahl von Patienten eine klinisch äußerst relevante Fragestellung hochkompetent bearbeitet. Die Ergebnisse decken sich mit den auch in Deutschland gemachten Beobachtungen und erlauben Patienten, die spätestens aus dem Internet über ihre deutlich eingeschränkte Lebenserwartung informiert werden, eine seriösere Prognose bezüglich ihrer Lebenserwartung zu geben. Einzige Schwäche der Studie ist ihre retrospektive Natur, wobei bekanntermaßen derzeit prospektive Studien durchgeführt werden. Bemerkenswert ist letzten Endes, dass bei allen neuropathologisch untersuchten Patienten die Verdachtsdiagnose MSA bestätigt werden konnte. 